\title{
REASONS FOR THE WEAK CORRELATION BETWEEN PROSTATE VOLUME AND URETHRAL RESISTANCE PARAMETERS IN PATIENTS WITH PROSTATISM
}

\author{
J. L. H. RUUD BOSCH, RIES KRANSE, RON VAN MASTRIGT AND FRITZ H. SCHRODER \\ From the Department of Urology, Erasmus University and Academic Hospital, Rotterdam, The Netherlands
}

\section{ABSTRACT}

In an attempt to increase our understanding of the clinical syndrome of benign prostatic hyperplasia (BPH) an analysis was made of the association between prostate volume as measured by transrectal ultrasound and several reported urodynamically determined urethral resistance parameters. Two types of obstruction can be recognized on the basis of urodynamic data: a compressive type characterized by a high urethral opening pressure and a prolonged isovolumetric contraction phase before urine flow can start, and a constrictive type characterized by a normal opening pressure and an increased slope of the urethral resistance relation. A combination of both types is often seen in BPH.

In our study, parameters that selectively quantify compression correlate weakly to moderately with prostate volume, whereas parameters that mainly quantify constriction do not correlate at all with prostate volume. Parameters that combine a measure for compression and constriction correlate less well with prostate volume than parameters that mainly quantify compression. The variation in prostate volume was found to determine the variation in urethral resistance by $15 \%$ or less depending on the parameter used, which implies that the different pathophysiological mechanisms that can increase urethral resistance in the complex process of clinical BPH are mainly determined by factors other than the volume of the prostate. Thus, despite the lack of correlation between prostate volume and urethral resistance, pressure-flow studies and the determination of urethral resistance parameters provide a valuable contribution to the understanding of the pathophysiology of voiding dysfunction in men with symptoms of prostatism.

\section{KEY WORDS: prostate, prostatic hypertrophy, urodynamics, urethra}

The clinical syndrome of benign prostatic hyperplasia (BPH) has been characterized by a combination of 3 properties: the presence of symptoms of prostatism, increased prostate volume and the presence of bladder outflow obstruction. ${ }^{1}$ The relationship among these properties is complex and only partially understood. Many patients, especially those with predominantly stromal hyperplasia, have small prostate volumes $^{2}$ and up to $34 \%$ with clinical BPH who are treated by transurethral resection of the prostate may be unobstructed urodynamically. ${ }^{3}$ Therefore, it is clear that a considerable number of those men who presently undergo transurethral resection of the prostate because of clinical BPH do not exhibit the combination of the aforementioned 3 properties. A reason for this is the fact that there is no general agreement about a clinical case definition of BPH due to the lack of a strong correlation among the symptoms with which a patient presents to a urologist, the presence of BPH in a histopathological sense, prostate volume and the presence of urodynamically proved bladder outflow obstruction.

Patients with bothersome symptoms of prostatism seek treatment because they would like to be relieved of these symptoms. Therefore, relief of symptoms is undoubtedly the best indicator for a successful treatment from the patient perspective. However, symptoms of prostatism are nonspecific, seem to be equally severe in age-matched groups of men and women, ${ }^{4}$ may at least to some extent be related to aging ${ }^{4}$ and have been shown to fluctuate considerably with time. ${ }^{5}$ This fluctuation is also evident in the placebo arms of 2 different randomized drug trials studying an $\alpha$-blocker and a $5 \alpha$-reductase inhibitor with a followup of 4 weeks and 1 year, respectively. ${ }^{6,7}$ Although symptom scores decreased significantly in the placebo groups in both studies, there was no

Accepted for publication August 19, 1994. significant change in urethral resistance parameters showing the reproducibility of pressure-flow studies. Furthermore, up to $30 \%$ of patients with prostatism followed for 5 years without being treated have shown symptomatic improvement. ${ }^{8}$ McGuire stated that symptoms of prostatism may be due to BPH but that BPH and these symptoms are not synonymous with bladder outflow obstruction. ${ }^{9}$ Furthermore, he stated that BPH cannot solely be defined by its response to a treatment, when the rate of spontaneous improvement is high enough to account for considerable improvement without treatment.

Future research should provide a better understanding of the origin of the symptoms that have traditionally been called the symptoms of prostatism. Until that time, the study of objective anatomical and physiological parameters related to $\mathrm{BPH}$ can be expected to provide the clearest insight into the natural history and pathophysiology of the disease. Because most urologists expect prostatectomy to relieve bladder outflow obstruction by the removal of a certain volume of obstructive prostatic tissue, the relationship between prostate volume and bladder outflow obstruction needs further clarification to increase our understanding of the pathophysiology of this disease.

\section{MATERIAL AND METHODS}

We studied 67 consecutive patients (mean age 66 years, range 37 to 84 ) who consented to participate in various treatment trials and who underwent detailed urodynamic studies. The patients were selected on the basis of symptoms of prostatism and a flow rate of less than $15 \mathrm{ml}$. per second.

Symptoms of prostatism. All patients complained of a weak stream with varying degrees of hesitancy, intermittency, urge-frequency, nocturia, post-void dribbling and/or a feeling 
of incomplete emptying. In most men symptoms were not scored according to one of the well known scoring systems but, after its introduction, the American Urological Association-7 index ${ }^{10}$ was determined in the last third of the patients for an average score of 19 (range 5 to 30 ). Patients with a proved or suspected neurogenic cause of the voiding dysfunction and those with prostatic or bladder cancer, or a urethral stricture were excluded.

Prostate volume. Transrectal ultrasound was performed using a $7 \mathrm{MHz}$. multiplane sector scanning probe (Bruel \& Kjaer). The planimetric technique of prostate volume measurement was used. ${ }^{11}$

Urodynamics. In all patients urodynamic studies were done, including 2 pressure-flow studies. The methods, definitions and units used were in accordance with the standards recommended by the International Continence Society. ${ }^{12}$ The use of urethral resistance parameters is an exception to this rule. The urodynamic examination involved 2 bladder fillings at a medium rate with fluid at room temperature. The bladder was emptied by catheterization. Thereafter, $2,5 \mathrm{~F}$ catheters were introduced: 1 was used for filling and 1 for pressure recording. During filling the pressure and volume in the bladder, and the pressure in the rectum were measured. During micturition the pressures in the bladder and rectum, flow rate and voided volume were measured with external pressure transducers and a Dantec flowmeter. The residual urine at the end of the examination was determined by catheterization or calculation. Throughout the study pelvic floor electromyography was recorded by stick-on electrodes and was used to indicate whether the patients were relaxing the pelvic floor muscles during voiding. From the 2 filling/voiding studies in each patient, the pressure-flow study with the highest maximum flow rate was used for the analysis. Pressure and flow rate signals were digitally stored with a specially developed computer program at a sample rate of $10 \mathrm{~Hz}$.

Parameters studied. After filtering the data using a low pass digital Butterworth filter with a cutoff frequency of 1 $\mathrm{Hz}$, pressure-flow plots were constructed from the stored detrusor pressure and flow rate signals. A flow delay time correction of 0.8 seconds was applied. A computer algorithm selected those points with a flow rate (Q) of greater than 0.25 ml. per second that fell within a $10 \mathrm{~cm}$. water band of the lowest monotonically increasing part of the pressure-flow plot. Through these selected points the passive urethral resistance relation ${ }^{13}$ (minimal urethral opening pressure + curvature of passive urethral resistance relation $Q^{2}$ ) and an orthogonal polynomial ${ }^{14}$ (average height of pressure-flow plot + average slope of pressure-flow plot [Q- $\beta]$ ) were fitted. In these formulas minimal urethral opening pressure ${ }^{13}$ is an estimate of the minimal urethral opening pressure, curvature of passive urethral resistance relation ${ }^{15}$ is an estimate of the curvature of the passive urethral resistance relation, average height of pressure-flow plot is an estimate of the average height and average slope of pressure-flow plot is an estimate of the average slope of the lowest part of the pressure-flow plot. $\beta$ is the average of the flow rate values that correspond to the data points that constitute the lowest part of the pressure-flow plot and roughly equals maximum flow rate divided by 2 .

Several other indexes for bladder outflow obstruction that yield values on a continuous scale were determined as well. Because of its wide use, the maximum flow rate is included in this study as an objectively determined urodynamic parameter. A poor flow rate may, however, be caused by detrusor failure and not by increased urethral resistance. The detrusor pressure at maximum flow also was determined. The value of minimal resistance, ${ }^{16}$ represented by the formula, detrusor pressure at maximum flow rate/maximum flow rate $^{2}$, changes whenever the flow rate changes (for example at different bladder volumes) even if there is no real change in urethral resistance. ${ }^{17} \mathrm{~A}$ group specific urethral resistance factor based on a statistical approximation of the average pressure-flow relationships measured in a large number of patients was determined. This parameter can be calculated for any micturition in which the maximum flow rate and corresponding detrusor pressure are known. ${ }^{18} \mathrm{OBI}$ is a parameter calculated as the weighted sum of the average height (A) and the average slope (B) of the lowest part of the pressure-flow plot, by means of the formula, $\mathrm{OBI}=\mathrm{A}+$ $2.4^{*} \mathrm{~B}$. The weighting factor 2.4 was obtained by Fisher's linear discriminant method. ${ }^{19}$

Statistical analysis. Using a statistical package, descriptive statistics, the Spearman rank correlation coefficient (r) and the coefficient of determination $\left(r^{2}\right)$ were determined to describe the association between prostate size and the various urodynamic parameters. The level of statistical significance was set at $p<0.05$ (1-tailed).

\section{RESULTS}

Descriptive statistics with respect to patient age, prostate volume and urodynamic parameters are summarized in table 1. Results of urodynamic studies before and after transurethral resection of the prostate have shown that a decrease in urethral resistance hardly ever occurs below a certain preoperative cutoff value. Such a cutoff value can, therefore, be chosen to separate patients with and without obstruction preoperatively. The rather low value of $20 \mathrm{~cm}$. water has been suggested as a cutoff point below which a patient is clearly considered not to have obstruction for minimal urethral opening pressure and patients with values of 20 to 30 $\mathrm{cm}$. water have been considered to have mild obstruction. ${ }^{13}$ For urethral resistance factor a cutoff value of $29 \mathrm{~cm}$. water has been suggested. ${ }^{2}$ However, it should be realized that values of 28 and $32 \mathrm{~cm}$. water, for example, represent only small differences in urethral resistance so that these cutoff points should be used cautiously.

To make statements about the relative contribution of prostate volume to urethral resistance in patients with prostatism, the study population should include a sufficient number of men with a normal and increased prostate volume, and a sufficient number with and without obstruction. The average values of the parameters minimal urethral opening pressure and urethral resistance factor for the patients included in this evaluation were in the obstructed range if cutoff values of 20 and $29 \mathrm{~cm}$. water are used, respectively (table 1). If a cutoff value of $29 \mathrm{~cm}$. water would have been chosen for urethral resistance factor and minimal urethral opening pressure, these parameters agreed that 13 of the 67 men (19\%) did not have obstruction. When using a rather low cutoff value, for example $20 \mathrm{~cm}$. water, for both parameters they agreed that 59 men did and 2 clearly did not have obstruction. Two patients had obstruction according to minimal urethral opening pressure but not according to urethral resistance factor, whereas 4 had obstruction according to urethral resistance factor but not according to minimal urethral opening pressure. If the urethral resistance factor, with

TABLE 1. Descriptive statistics of the patients showing mean values and ranges for the different urodynamic parameters, which were used in the correlation studies with prostate volume

\begin{tabular}{lcr}
\hline \multicolumn{1}{c}{ Parameter (unit) } & \multicolumn{2}{c}{ Mean (range) } \\
\hline Age (yrs.) & 66 & $(37-84)$ \\
Prostate vol. (cm. ${ }^{3}$ ) & 46 & $(8-132)$ \\
Maximum flow rate (ml./sec.) & 6.6 & $(1.5-12.6)$ \\
Detrusor pressure at maximum flow rate (cm. water) & 61 & $(25-127)$ \\
Minimal resistance (cm. water $\times$ sec. $^{2} / \mathrm{ml}^{2}$ ) & 2.4 & $(0.2-26.1)$ \\
Minimal urethral opening pressure (cm. water) & 40 & $(11-78)$ \\
Curvature of passive urethral resistance relation & $0.85(0.52-7.84)$ \\
$\quad$ (cm. water $\times$ sec. ${ }^{2} /$ ml. $^{2}$ ) & & \\
Urethral resistance factor (cm. water) & 38 & $(15-77)$ \\
Av. height of pressure-flow plot (cm. water) & 48 & $(15-87)$ \\
Av. slope of pressure-flow plot (cm. water/ml./sec.) & 4.7 & $(0.6-17.7)$ \\
OBI (dimensionless) & 60 & $(17-107)$ \\
\hline
\end{tabular}


its cutoff value of $29 \mathrm{~cm}$. water, is used as the only classifier, then 20 of 67 patients ( $30 \%$ ) are classified as urodynamically unobstructed cases. In this population of men, who were selected for treatment on the basis of symptoms of prostatism and a maximum flow rate of less than $15 \mathrm{ml}$. per second, up to $30 \%$, therefore, did not have urodynamic evidence of obstruction. This percentage corresponds to data of Abrams et al, who found that $36 \%$ of 318 patients with symptoms suggestive of outflow obstruction did not have obstructed outflow urodynamically $^{20}$ and to data of Rollema and van Mastrigt, ${ }^{3}$ who found that up to $34 \%$ of cases of clinical BPH treated by transurethral resection of the prostate may be unobstructed urodynamically.

The range of prostate volumes in our patients is wider than the range found in a community-based sample of men 55 to 74 years old ${ }^{21}$ of our patients $12 \%$ had a prostate volume of less than $20 \mathrm{~cm}^{3}$, compared to only $5 \%$ of the men in the community-based sample. Furthermore, $67 \%$ of our men had a prostate volume of greater than $30 \mathrm{~cm}^{3}$, while this was the case in $60 \%$ of the community-based men.

The coefficients of correlation between the different parameters and prostate volume are summarized in table 2 . The coefficients of determination show that the variation in the values of the parameters used to characterize bladder outflow obstruction can be attributed to the variation in prostatic volume by only $15 \%$ or less depending on the parameter selected. In descending order, the best (but still moderate) correlations between prostate volume and the parameters studied are found for average height of pressure-flow plot ( $r=0.39, p=0.001$, figure), minimal urethral opening pressure $(r=0.38, p=0.001)$, OBI $(r=0.31, p=0.006)$ and detrusor pressure at maximum flow rate $(\mathrm{r}=0.30, \mathrm{p}=$ 0.006). The parameters average height of pressure-flow plot, minimal urethral opening pressure, detrusor pressure at maximum flow rate and urethral resistance factor correlate better with prostate volume than average slope of pressureflow plot and curvature of passive urethral resistance relation, which both show a statistically nonsignificant correlation with prostate volume. The parameter OBI, which combines height and average slope of pressure-flow plot, correlates less well with prostate volume than average height of pressure-flow plot alone. Maximum flow rate and minimal resistance show a statistically nonsignificant correlation with prostate volume.

\section{DISCUSSION}

The properties of parameters to characterize urethral resistance are the subject of ongoing discussions. From a physical viewpoint it is clear that pressure-flow studies or parameters derived from them are a more appropriate indicator of urethral resistance than uroflowmetry alone. Despite this, the urological community has been reluctant to accept the inclusion of more sophisticated urodynamics in the diagnos-

TABLE 2. Correlation between prostate volume and various parameters that characterize bladder outflow obstruction represented by Spearman correlation coefficients ( $r$ ) and coefficients of determination $\left(r^{2}\right)$

\begin{tabular}{lccc}
\hline \multicolumn{1}{c}{ Parameter } & $\mathrm{r}$ & $\mathrm{r}^{2}$ & $\mathrm{p}$ Value \\
\hline Maximum flow rate & -0.05 & -0.003 & $0.33^{*}$ \\
Detrusor pressure at maximum flow & 0.30 & 0.09 & 0.006 \\
$\quad$ rate & 0.18 & 0.03 & $0.07^{*}$ \\
Minimal resistance & 0.38 & 0.14 & 0.001 \\
Minimal urethral opening pressure & 0.08 & 0.006 & $0.26^{*}$ \\
Curvature of passive urethral & & & \\
$\quad$ resistance relation & 0.24 & 0.06 & 0.027 \\
Urethral resistance factor & 0.39 & 0.15 & 0.001 \\
Av. height of pressure flow plot & 0.04 & 0.002 & $0.37^{*}$ \\
Av. slope of pressure flow plot & 0.31 & 0.10 & 0.006 \\
OBI & & &
\end{tabular}

* Not statistically significant.

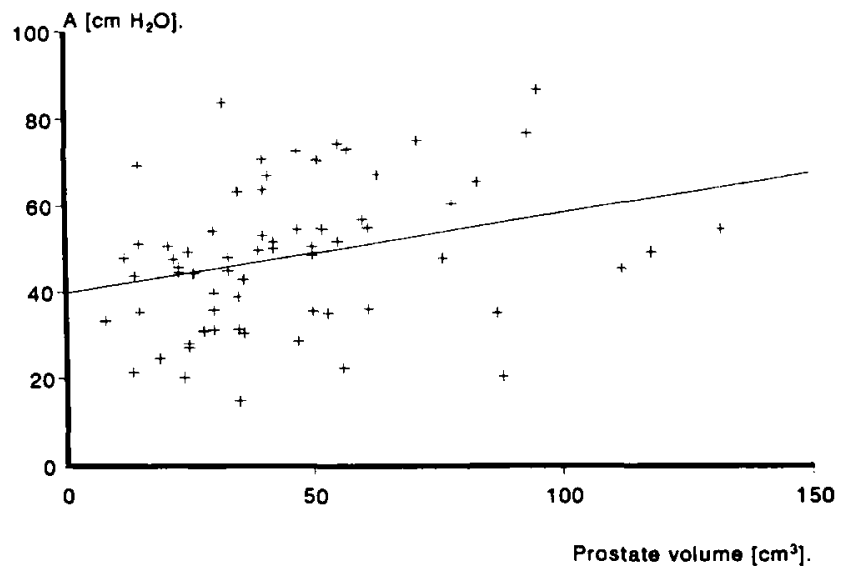

Scattergram with regression line shows correlation between urethral resistance parameter $A$ and prostate volume $(r=0.39, p=$ 0.001 ). Parameter $A$ is estimate of average height of lowest part of pressure-flow plot.

tic evaluation of patients with symptoms of prostatism. Some of the reasons for this reluctance are that urodynamic studies are invasive, there is a poor correlation between symptom severity or prostate size and simple urodynamic parameters, such as maximum flow rate and post-void residual urine volume, ${ }^{22}$ and the preoperative severity of urodynamically determined bladder outflow obstruction seems to be a moderate predictor of outcome as measured by subjective symptoms and flow rate. ${ }^{23}$ Also, many patients without urodynamic evidence of obstruction seem to do well symptomatically after prostatectomy. ${ }^{23}$ A correlation between symptoms and urodynamically proved outflow obstruction has been shown in 1 study only, and only for the symptoms of weak stream and hesitancy. ${ }^{24}$ Finally, the correlation between prostate volume and urodynamically determined urethral resistance is believed to be weak.

Our study was performed to clarify the latter point, that is the relationship between prostate volume and urethral resistance in patients with symptoms of prostatism. The noncorrelation among prostate volume, and the parameters maximum flow rate and minimal resistance in our evaluation is in agreement with the fact that pressure-flow studies or parameters derived from them are a more appropriate indicator of urethral resistance than uroflowmetry alone or a parameter that is biased by changes in flow rate, such as minimal resistance. These results are at variance with those of other investigators who noted a strong correlation $(r=0.8$, $\mathrm{p}<0.001$ ) between prostate volume and the parameter minimal resistance. ${ }^{25}$ However, the patients in the latter study were highly select, since only men with voiding pressures of greater than $100 \mathrm{~cm}$. water and with flow rates of less than $15 \mathrm{ml}$. per second were included. Tan et al reported a weak correlation between minimal urethral opening pressure and prostatic volume $(r=0.27, p=0.003)$ in $118 \mathrm{BPH}$ patients with the same average age as our men. ${ }^{26}$ Details about the prostatic volumes of those patients were not given.

In our study the coefficient of determination was 0.15 at best (for the parameter average height of pressure-flow plot), which indicates that the variation in urethral resistance is determined by the variation in prostate volume by only $15 \%$ or less. Therefore, it can be concluded that most of the urethral resistance is determined by factors other than prostate volume alone. Since the correlation between prostate volume and urethral resistance is relatively poor, the size of the prostate should not be an important consideration when determining the necessity for therapy. The choice of the treatment modality, however, depends more on prostate volume. 
Two types of obstruction may be recognized on the basis of urodynamic data: a compressive type characterized by a high urethral opening pressure and a prolonged isovolumetric contraction phase before flow can start, and a constrictive type characterized by a normal opening pressure and an increased slope of the passive urethral resistance relation. ${ }^{13}$ In most BPH patients the compressive obstruction is accompanied by a certain degree of constrictive obstruction. In the urethral resistance factor the constrictive and compressive elements are combined in 1 parameter based on a statistical approximation of the average pressure-flow relationship measured in a large number of patients. ${ }^{18}$ Selective quantifiers for compression, that is the parameters average height of pressure-flow plot and minimal urethral opening pressure, correlate better with prostate volume than the quantifiers for constriction, that is average slope of pressure-flow plot and curvature of passive urethral resistance relation, which both show a statistically nonsignificant correlation with prostate volume. The parameters $\mathrm{OBI}$, detrusor pressure at maximum flow rate and urethral resistance factor, which combine the compressive and constrictive factor, correlate less well with volume than average height of pressure-flow plot and minimal urethral opening pressure.

The weak to moderate correlation between prostate volume and various parameters describing bladder outflow obstruction does not disqualify these parameters and make them less useful in the characterization of voiding dysfunction due to BPH. From a pathophysiological viewpoint, both factors are important in the characterization of voiding dysfunction and, therefore, a parameter combining both may describe the global dysfunction more accurately. The histological properties of the gland may at least partly determine the type of obstruction. Not all prostates treated by transurethral resection of the prostate show the same histological abnormalities. Dorflinger et al showed that among 81 patients predominantly stromal hyperplasia, predominantly glandular hyperplasia and mixed hyperplasia were present in 48,28 and $23 \%$, respectively. ${ }^{2}$ Although symptomatically there were no differences in outcome, the men with predominantly stromal hyperplasia had smaller resected weights and a significantly lower maximum flow rate 3 months postoperatively. They concluded that the stromal group may have incomplete relief of obstruction with standard transurethral resection of the prostate, which conserves the surgical capsule, and they may be prone to suffer early recurrence of symptoms. This finding indicates that some treatment options may have a more pronounced effect on 1 or both aspects of bladder outflow obstruction. $\alpha$-Blocker treatment has a relaxing effect on smooth muscle cells and, therefore, can theoretically influence the elasticity of the prostatic urethra. Urodynamic effects of $\alpha$-blocker treatment can be expected to be more clear when a parameter that emphasizes or includes the factor of constriction is used. Urethral resistance factor and OBI were able to show small but significant effects of treatment with the $\alpha$-blocker doxazosin at a dose of 2 and $4 \mathrm{mg} .{ }^{19} \mathrm{~A}$ study of the urodynamic effects of transurethral microwave thermotherapy has shown no decrease in minimal urethral opening pressure. However, a decrease in the curvature of the passive urethral resistance relation (lower value of curvature of passive urethral resistance relation) was noted with this treatment modality and it was postulated that urethral elasticity changes with transurethral microwave therapy. ${ }^{27}$

In conclusion, the correlation between prostate volume and parameters for bladder outflow obstruction is at best only moderate, which does not imply that these parameters are of limited value. Some parameters are better suited to study 1 of the 2 elements of obstruction, that is either compression or constriction. A particular treatment modality may have more pronounced effects on 1 of these 2 elements. Furthermore, the different pathophysiological mechanisms that can increase urethral resistance in the complex process of clinical
$\mathrm{BPH}$ are mainly determined by factors other than the volume of the prostate. Thus, despite the lack of correlation between prostate volume and urethral resistance, pressure-flow studies and the determination of urethral resistance parameters provide a valuable contribution to the understanding of the pathophysiology of voiding dysfunction in men with symptoms of prostatism.

\section{REFERENCES}

1. Hald, T.: Urodynamics in benign prostatic hyperplasia: a survey. Prostate, suppl., 2: 69, 1989.

2. Dørflinger, T., England, D. M., Madsen, P. O. and Bruskewitz, R. C.: Urodynamic and histological correlates of benign prostatic hyperplasia. J. Urol., 140: 1487, 1988.

3. Rollema, H. J. and van Mastrigt, R.: Improved indication and followup in transurethral resection of the prostate using the computer program CLIM: a prospective study. J. Urol., 148: $111,1992$.

4. Lepor, H. and Machi, G.: Comparison of AUA symptom index in unselected males and females between fifty-five and seventynine years of age. Urology, 42: 36, 1993.

5. Diokno, A. C., Brown, M. B., Goldstein, N. and Herzog, A. R.: Epidemiology of bladder emptying symptoms in elderly men. J. Urol., 148: 1817, 1992.

6. Rollema, H. J., Rosier, P., Janknegt, R. A. and van Mastrigt, R.: Efficacy of alpha-blocker (Doxazosin) in BPH appraised by pressure-flow (CLIM) analysis. Neurourol. Urodynam., 10: $295,1991$.

7. Rollema, H. J., Rosier, P. F. W. M., van Mastrigt, R. and Janknegt, R. A.: Clinical efficacy of Proscar (MK 906) in BPH, objectively appraised by pressure-flow measurements analyzed with the computer program $\mathrm{Dx} / \mathrm{Clim}$; 2 years results. Neurourol. Urodynam., 11: 392, abstract 60, 1992.

8. Ball, A. J., Feneley, R. C. and Abrams, P. H.: The natural history of untreated "prostatism." Brit. J. Urol., 53: 613, 1981.

9. McGuire, E. J.: The role of urodynamic investigation in the assessment of benign prostatic hypertrophy. J. Urol., 148: $1133,1992$.

10. Barry, M. J., Fowler, F. J., Jr., O'Leary, M. P., Bruskewitz, R. C., Holtgrewe, H. L., Mebust, W. K. Cockett, A. T. K. and the Measurement Committee of the American Urological Association: The American Urological Association symptom index for benign prostatic hyperplasia. J. Urol., 148: 1549, 1992.

11. Torp-Pedersen, S., Juul, N. and Jakobsen, H.: Transrectal prostatic ultrasonography. Equipment, normal findings, benign hyperplasia and cancer. Scand. J. Urol. Nephrol., suppl., 107: $19,1988$.

12. Abrams, P., Blaivas, J. G., Stanton, S. L. and Andersen, J. T.: Standardization of terminology of lower urinary tract function. In: Clinical Neuro-Urology, 2nd ed. Edited by R. J. Krane and M. B. Siroky. Boston: Little, Brown \& Co., p. 651, 1991.

13. Schäfer, W.: Principles and clinical application of advanced urodynamic analysis of voiding function. Urol. Clin. N. Amer., 17; $553,1990$.

14. Kranse, M. and van Mastrigt, R.: Fitting orthogonal polynomials to the lowest part of a pressure flow plot. Neurourol. Urodynam., 10: 290, abstract 7, 1991.

15. Schäfer, W.: Urethral resistance? Urodynamic concepts of physiological and pathological bladder outlet function during voiding. Neurourol. Urodynam., 4: 161, 1985.

16. Frimødt-Möller, C. and Hald, T.: Clinical urodynamics. Methods and results. Scand. J. Urol. Nephrol., suppl. 15, 6: 143, 1972.

17. Bosch, R. J. L. H., Griffiths, D. J., Blom, J. H. M. and Schröder, F. H.: Treatment of benign prostatic hyperplasia by androgen deprivation: effects on prostatic size and urodynamic parameters. J. Urol., 141: 68, 1989.

18. Griffiths, D. J., van Mastrigt, R. and Bosch, R.: Quantification of urethral resistance and bladder function during voiding, with special reference to the effects of prostate size reduction on urethral obstruction due to benign prostatic hyperplasia. Neurourol. Urodynam., 8: 17, 1989.

19. van Mastrigt, $R$. and Kranse, M.: Automated evaluation of urethral obstruction. Urology, 42: 216, 1993.

20. Abrams, P. H. and Feneley, R. C. L.: The significance of the symptoms associated with bladder outflow obstruction. Urol. Int., 33: 171, 1978. 
21. Bosch, J. L. H. R., Hop, W. C. J., Niemer, A. Q. H. J., Bangma, C. H., Kirkels, W. J. and Schröder, F. H.: Parameters of prostate volume and shape in a community-based population of men 55 to 74 years old. J. Urol., 152: 1501, 1994.

22. Barry, M. J., Cockett, A. T. K., Holtgrewe, H. L., McConnell, J. D., Sihelnik, S. A. and Winfield, H. N.: Relationship of symptoms of prostatism to commonly used physiological and anatomical measures of the severity of benign prostatic hyperplasia. J. Urol., 150: 351, 1993.

23. Neal, D. E., Ramsden, P. D., Sharples, L., Smith, A., Powell, P. H., Styles, R. A. and Webb, R. J.: Outcome of elective prostatectomy. Brit. Med. J., 299: 762, 1989.

24. Abrams, P. H. and Feneley, R. C. L.: The significance of the symptoms associated with bladder outflow obstruction. Urol. Int., 33: 171, 1978.
25. Kadow, C., Abrams, P. H. and Penry, J. B.: The relationship between urodynamic parameters of outflow obstruction and prostatic volume in men with prostatism. Proceedings of the 14th Annual Meeting of the International Continence Society, Innsbruck, Austria, p. 125, September 13-15, 1984.

26. Tan, H. K., Höfner, K., Kramer, A. E. J. L., Thon, W. F., Grünewald, V. and Jonas, U.: Benign prostatic hypertrophy: prostatic size, obstruction parameters, detrusor contractility and their interdependence. Neurourol. Urodynam., 12: 412, 1993.

27. Höfner, K., Tan, H.-K., Kramer, A. E. J. L., Kuczyk, M., von Dalwig-Nolda, D. and Jonas, U.: Changes in outflow obstruction in patients with benign prostatic hypertrophy after transurethral microwave thermotherapy. Neurourol. Urodynam., 12: 376, 1993. 